\title{
Keeping up with the genomes: efficient learning of our increasing knowledge of the tree of life
}

\author{
Zhengqiao Zhao', Alexandru Cristian² and Gail Rosen ${ }^{1 *}$ (1)
}

\author{
*Correspondence: glr26@drexel.edu \\ ${ }^{1}$ Ecological and Evolutionary \\ Signal-process and Informatics \\ (EESI) Lab, Department of Electrical \\ and Computer Engineering, Drexel \\ University, Market Street, \\ Philadelphia, US \\ Full list of author information is \\ available at the end of the article
}

\begin{abstract}
Background: It is a computational challenge for current metagenomic classifiers to keep up with the pace of training data generated from genome sequencing projects, such as the exponentially-growing NCBI RefSeq bacterial genome database. When new reference sequences are added to training data, statically trained classifiers must be rerun on all data, resulting in a highly inefficient process. The rich literature of "incremental learning" addresses the need to update an existing classifier to accommodate new data without sacrificing much accuracy compared to retraining the classifier with all data.
\end{abstract}

Results: We demonstrate how classification improves over time by incrementally training a classifier on progressive RefSeq snapshots and testing it on: (a) all known current genomes (as a ground truth set) and (b) a real experimental metagenomic gut sample. We demonstrate that as a classifier model's knowledge of genomes grows, classification accuracy increases. The proof-of-concept naïve Bayes implementation, when updated yearly, now runs in $1 / 4^{\text {th }}$ of the non-incremental time with no accuracy loss.

Conclusions: It is evident that classification improves by having the most current knowledge at its disposal. Therefore, it is of utmost importance to make classifiers computationally tractable to keep up with the data deluge. The incremental learning classifier can be efficiently updated without the cost of reprocessing nor the access to the existing database and therefore save storage as well as computation resources.

Keywords: Incremental learning, Naïve Bayes taxanomic classifier, RefSeq, Metagenomics

\section{Background}

Recent advances in genomics have resulted in exponential increases in the rate at which data is collected. Inspired by Zynda [1], we visualize the growth of National Center for Biotechnology Information (NCBI) Reference Sequence (RefSeq) bacterial genome database [2,3] in Fig. 1. Figure 1a shows the total number of complete genomes

(c) The Author(s). 2020 Open Access This article is licensed under a Creative Commons Attribution 4.0 International License, which permits use, sharing, adaptation, distribution and reproduction in any medium or format, as long as you give appropriate credit to the original author(s) and the source, provide a link to the Creative Commons licence, and indicate if changes were made. The images or other third party material in this article are included in the article's Creative Commons licence, unless indicated otherwise in a credit line to the material. If material is not included in the article's Creative Commons licence and your intended use is not permitted by statutory regulation or exceeds the permitted use, you will need to obtain permission directly from the copyright holder. To view a copy of this licence, visit http://creativecommons.org/licenses/by/4.0/. The Creative Commons Public Domain Dedication waiver (http://creativecommons.org/publicdomain/zero/1.0/) applies to the data made available in this article, unless otherwise stated in a credit line to the data. 

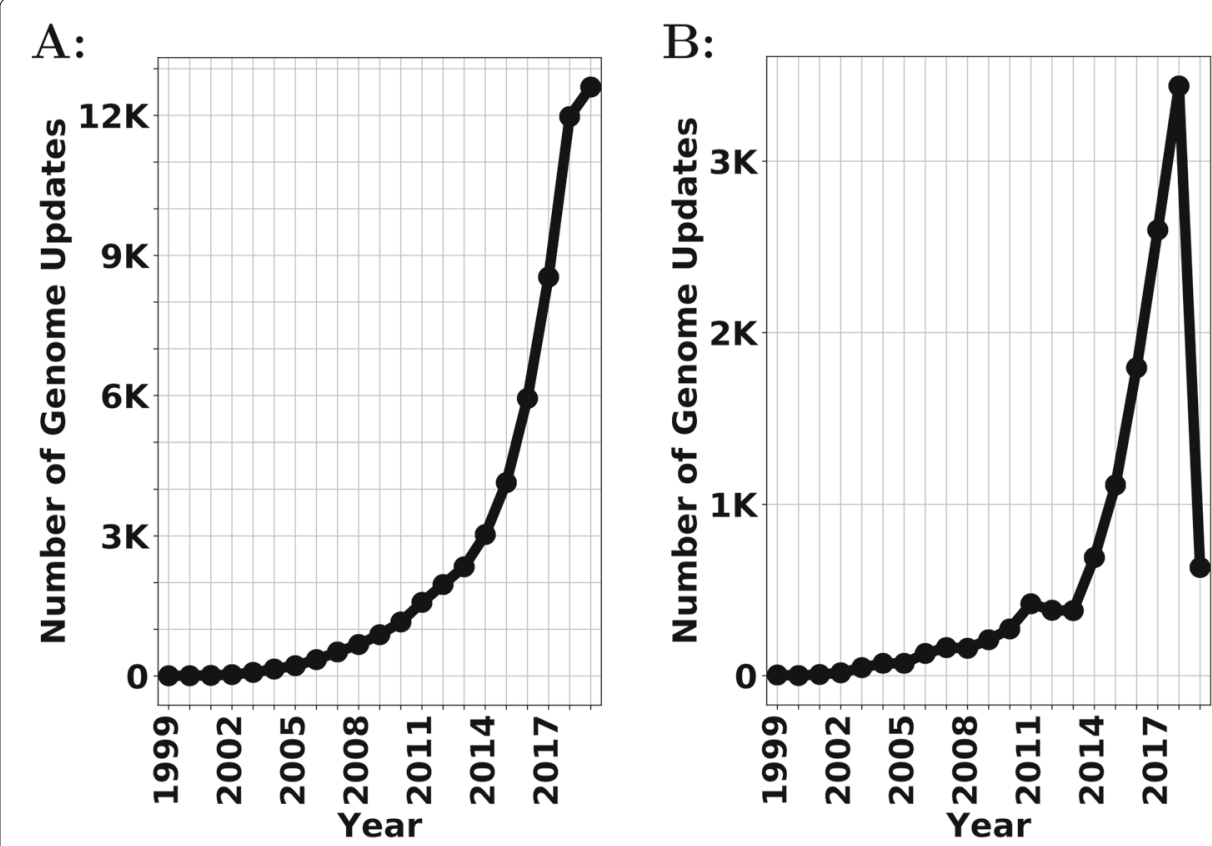

Fig. 1 Number of updates in the NCBI bacterial genomes from the RefSeq database a Accumulative number of genome updates per year; $\mathbf{b}$ compared with previous year, the number of new updates per year

added/updated in the database in yearly increments until March 2nd $2019^{1}$, and Fig. 1b shows the number of new and updated completed genomes every year. As shown in Fig. 1, there are now thousands of genomes being sequenced per year, providing vital information for understanding prokaryotic species diversity, with major efforts like the Genome Encyclopedia of Bacteria and Archaea contributing to this expansion [4].

Moreover, driven by advances in technology and significant reductions in the cost of analysis, microbiome research has unlocked a wealth of data in recent years [5]. In fact, the cost of sequencing DNA is reducing at a rate that is outpacing Moore's law [6]. In other words, the increase of computational power cannot keep up with the growth of the number of genomes (as well as metagenomes) being added.

One of the main challenges in metagenomics is to answer, "Who is there?": identifying the microorganisms in the sample. Taxonomic classification - classifying the reads in metagenome sequencing data - uses aligners, read mappers, classifiers and other "base techniques" to solve this problem [7-11]. Taxonomic classification is usually one of the first steps in a metagenomic pipeline [12]. Once these organisms are identified, they are then used in downstream analyses, such as alpha/beta diversity measures, ordination, feature selection, phenotype classification, etc.

However, while many methods have been proposed for taxonomic classification [13, 14], the accuracy of these methods using different training databases has not been fully tested. This is an important issue - because as new genome data is generated, training data sets, such as the commonly used NCBI Reference Sequence Database (RefSeq), will change over time. Consistency between databases and completeness of genomes is already an issue plaguing these databases [15]. Loeffler et al. highlight the cost of computational power and storage requirements of maintaining a master reference sequence

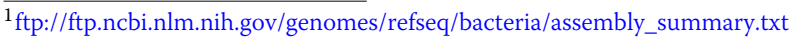


database, with a proposed solution of a "continuous assembly approach" (supported by the institution's infrastructure and the scientific community supplying the data sources) [15]. From a classifier perspective, Nasko et al. recently demonstrated that more reads are classified (as opposed to be assigned to an unclassified/unknown class) by the Kraken classifier with newer database versions [16]. Nasko's analysis also suggested that changes in RefSeq over time may influence but not necessarily reduce the misclassification rate, with genus/species false positives shown to be $1 \%$ and $8 \%$ respectively. These metrics were, however, calculated only on a selection of 10 genomes. Accordingly, given that there is an ongoing rapid expansion of genomic data of microbial diversity, it is imperative to update taxonomic classifiers as new genomes/genes are discovered. Simply failing to update the model will result in lower accuracy due to incomplete knowledge. In addition, the way that most researchers train their taxonomic classifiers is a static process: A fixed number of genomes are used as training data, and when more reference genomes are added to NCBI, the classifiers must be completely retrained to accommodate the new data. This type of training, however, is computationally unsustainable, due to the aforementioned fast expansion of Reference Sequence (RefSeq) database.

The traditional model of retraining of classifiers, each time training on the entire dataset, cannot sufficiently keep pace with new discoveries. One possible course of action would be to update the database less frequently. However, the resulting analysis will not be as "accurate" as it could be if frequently updated with knowledge about new genomes. As a result, there is a need for new innovations in updating classifiers. In RefSeq database, we can observe a new genome being added, removed and updated and its taxonomic labels can be merged with, changed to other labels and even removed from the database. In our work, we only focus on the cases where a new genome is added to the database. However, our algorithm can also be extended to handle the taxonomic nodes merging scenarios (see our discussion in "Incremental naïve Bayes classifier" section).

An intuitive solution is to update the training model for new data only, instead of reprocessing the whole database. In this way, we can greatly reduce the amount of redundant computations to compute more frequent updates [17]. The naïve Bayes classifier (NBC) is a natural solution, since only the columns (of the $k$-mer frequency table) of the classes being updated or added need to be changed and there are studies that have successfully utilized incremental learning naïve Bayes classifier for text classification [18-21]. We demonstrate that the incremental learning algorithm allows computers to "continually learn" new information seamlessly and alleviate the current inefficient and inadequate retraining practices. We also show that the limitation of naïve Bayes classifier: its classification results can be biased by species classes that have many genomic examples (i.e: well-represented species). In this paper, we show that incremental learning algorithm is a promising solution. We developed a proof-of-concept incremental implementation of a naïve Bayes classifier. We then show that the taxonomic classification task can benefit from learning incrementally from the NCBI dataset in respect to two aspects:

- the classifier gains accuracy over the time by constantly updating the classifier with the latest knowledge (genomes).

- the time of update can be greatly reduced by incremental learning.

While some of the results show that NBC might not be the best choice for taxonomic classifier, we hypothesize that other algorithms may be afflicted by this class 
bias as well (see our discussion in "Case example of B. aphidicola and C. botulinum misclassification" section). So, our work serves as a lesson.

Therefore, the main aim of this paper is to show how incremental learning can reduce computational time and enable constant improvements in accuracy through frequent updates over time. The paper highlights the importance of incremental learning and its promise, rather than the NBC classifier selection itself. Much future work remains to examine how other taxonomic classifiers can be "incrementalized".

In this paper, "incrementalized" version of naïve Bayes taxonomic classifier demonstrates how the computational time of training is reduced while still retaining the same accuracy as the original implementation [7]. To be specific, when a new labeled sequence is available, we update the model to learn new information: If the new sequence is from an existing class, then the class will be updated based on Bayes rule; otherwise, a new class is created to accommodate this new organism. Therefore, we do not have to create a new model from scratch and process the whole database. Instead, we only need to process the new data to update the model. In addition, we present an implementation of NBC that optimizes RAM and the number of cores via a smart loading scheme that is scalable for various architectures. Our implementation of NBC is open source here: https://github. com/EESI/Naive_Bayes. Our contribution in this paper is three-fold:

- show quantitative classification results that improve over time by training a classifier on progressive RefSeq snapshots and testing the classifier on simulated reads using 5-fold cross-validation.

- qualitatively demonstrate how the classification composition of a real metagenomic gut sample changes over time by training a classifier on RefSeq dataset in 5 different years.

- propose a proof-of-concept incremental implementation of Naïve Bayes taxonomic classifier $(\mathrm{NBC}++)$, that can be efficiently updated with new information without having to reprocess the existing database, which drastically reduces the training time of the classifier when new data is available compared to the training time of the non-incremental implementation.

\section{Related work}

Our work relates to both incremental learning and microbial taxonomic classification fields. We develop and evaluate an "incrementalized" version of the naïve Bayes taxonomic classifier [7].

\section{Related work on incremental learning}

In an incremental learning setting defined by Kochurov et al., a dataset $D=\left\{\mathbf{x}_{i}, y_{i}\right\}_{i=1}^{N}$ is divided into $T$ parts $D=\left\{D_{1}, \cdots, D_{T}\right\}$, which arrive sequentially [22]. The goal is to build an efficient algorithm that takes as input (a) a model, $\mathbf{M}_{t}$ that has been trained on the first $t$ units of data $\left\{D_{1}, \cdots, D_{t}\right\}$ and (b) new data, $D_{t+1}$, to then output an updated model $\mathbf{M}_{t+1}$. The efficiency of the algorithm can be achieved without direct access to the previous training data, $\left\{D_{1}, \cdots, D_{t}\right\}$, while performance is maintained without catastrophically forgetting the previous model [23]. Ensemble learning algorithms can be used to achieve incremental learning. Polikar et al. proposed an incremental learning algorithm, Learn++, that ensembles "weak" classifiers during the training [24]. New classifiers will be added to learn and explain the new and misclassified examples. Support Vector 
Machine (SVM) are "incrementalized" by many researchers [25-27]. SVM finds support vectors and determines a decision boundary that best separates the support vectors. A set of support vectors can be viewed as a good abstraction of training data, therefore, the existing training database can be compressed using support vectors. When the data is presented to the learning algorithm sequentially in batches, one can compress the data of the previous batches to their support vectors. Then, for each new batch of data, a SVM is trained on the new data and the support vectors from the previous learning step [25]. Deep learning approaches have been producing state-of-the-art results yet they continue to suffer from catastrophic forgetting, a dramatic decrease in overall performance when training with new data added incrementally [23]. Kochurov et al. utilize a Bayesian approach to update the deep learning model with new data [22]. Castro et al. uses new data and a small exemplar set from the old classes to update the model [23]. Both methods show improvement on performance for incremental deep learning models. The deep neural network by Kochurov et al. works for models that have a determined number of classes which remains the same over time [22] whereas the method proposed by Castro et al. handles the cases that novel classes can be available when training [23]. Typically, there are two types of incremental learning situations: 1 ) new training data $D_{t+1}$ contains data from existing classes only; 2) new training data $D_{t+1}$ has samples from both novel classes (new classes that were not known to the existing model) and existing classes [21]. In our application, since the amount of novel bacteria are continually and increasingly being sequenced (see Additional file 1 - The number of taxonomic labels per year), our incremental learning algorithm needs to handle the increasing amount of novel classes being added to the database as well.

\section{Related work on taxonomic classification}

$16 \mathrm{~S}$ ribosomal RNA is useful for placing organisms on the phylogenetic tree [28], and the widely-used Ribosomal Database project classifier [29] uses a naïve Bayes classifier to provide taxonomic assignments for microbial $16 \mathrm{~S}$ rRNA sequences. However, $16 \mathrm{~S}$ rRNA amplification is often insufficient for discrimination at the species and strain levels of classification [13] because $16 \mathrm{~S}$ rRNA genes are too slowly evolving to reliably separate the validly named species [30,31]. Whole-genome shotgun sequencing provides researchers' access to more genomic content of organisms and thus, can yield finer taxonomic resolution. Therefore, in this paper, we focus on metagenomic taxonomic classification that classifies metagenomic reads to species level taxa [7-9, 32].

The Basic local alignment search tool (Blast) [32] is one of best methods for assigning a taxonomic label to an unknown sequence $[8,13]$. Other Blast-based algorithms further improve the taxonomic classification accuracy by incorporating other information (e.g. last/lowest common ancestor) [33]. However, Blast is not designed for high-throughput metagenomic reads classification, and its computationally expensive to get local alignments for hundreds of thousands and millions of reads. Other alignmentbased techniques can be mapping-based - using methods like the Burrows-Wheeler transform (BWT) or variants of hash tables [11,34]. While enabling fast queries, mapping tools need to spend "training time" to compress/prefilter the reference database. Complementary to alignment-based techniques, there are $k$-mer composition based tools. Hash tables have proven to be successful for these alignment-free techniques as well $[8,35]$, and query searches are 909 times faster than Megablast (a fast BLAST program) [8]. To achieve 
the fast query times, construction of such efficient hashing model can be a computational upfront cost and therefore is subject to the longer build times when the reference database expands. While the "training" is done offline so that queries benefit from the quick turnaround time, such training could become cumbersome if new queries must use an old database and wait for the read mapper to be updated with new reference genomes.

In an incremental learning scenario, when new genomes are added to the reference database, the blast database should be updated to accommodate the new genomes. Currently, Blast databases are updated daily ${ }^{2}$. However, traditionally, Blast is not designed for high-throughput metagenomic reads classification, and its expensive to compute local alignments for hundreds of thousands and millions of reads. Mapping and $k$-mer classifier tools need to build BWT/hash indexes each time on a complete "set", which will take longer as the database size grows, and versions, that do not permit incremental updates, have not been implemented yet. For example, Kraken [8] uses exact-match of $k$-mers, rather than inexact alignment of sequences to perform taxonomic classification. The classification is based on a well organized $k$-mer to lowest common ancestor (LCA) mapping, and it is very efficient to search $k$-mers in the database. The authors show that Kraken's accuracy is comparable to Blast based sequence classifiers and run faster than those competing programs. When there are new genomes added to the database, Kraken needs to be trained from scratch. Although Kraken is not designed for quick updates, it is potentially "incrementalizable": only a fraction of the taxonomy tree (the nodes of updated/newly added genomes and their ancestral nodes), and the Kraken $k$-mer database needs to be updated. The taxonomy tree in Kraken is used for the lowest common ancestor (LCA) computation and keeping track of which $k$-mers are unique to certain clades for efficient $k$-mer to taxa assignment. Thus, both the tree and which $k$-mers are unique to each clade must be updated for newly added genomes. Clever ways of deciding which areas to update need to be developed.

NBC [7] is a supervised machine learning based approach for taxonomic classification. It uses a naïve Bayes classifier (NBC) to classify all metagenomic reads to their best taxonomic match and is good at estimating the number of reads of particular organisms that are known to the classifier shown via $l 1$-norm distance and log-modulus deviation [13]. The NBC classifier is, by nature, "incrementalizable". When new genomes are added, the classifier can be updated with only the conditional probabilities of the new species. In recent years, there are novel tools that incorporate deep learning techniques to perform taxonomic classification [36-38]. However, the deep learning models can't be quickly updated in case of new species. The final dense layer has to be modified to accommodate the new species. The update speed can be improved by training the new model with weight from the existing deep learning model. In this paper, we "incrementalize" the NBC classifier to show that we can use less computation while retaining the same accuracy and demonstrate the efficacy of our implementation over increasing knowledge.

\section{Results}

In this section, we use the NCBI Reference Sequence (RefSeq) bacterial genome database to evaluate: 1) the k-mer size influence on taxonomic classification accuracy; 2) the incremental taxonomic classification performance; 3 ) the dynamics of a classifier trained on

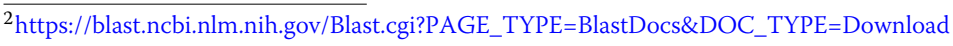


different yearly snapshots of the RefSeq bacterial database and its influence on taxonomic profiling results. These results demonstrate the necessity of incremental learning for metagenomic taxonomic classification.

It is important to note that unlike the original implementation of NBC [7], where each read was first classified on the genome-level, each read here is classified to a species class. All genomes' $k$-mers within a species are aggregated across strain genomes and trained to be a "species class".

\section{Evaluating NBC on a larger dataset: $\boldsymbol{k}$-mer size vs. accuracy}

Benefiting from the incremental learning ability, we don't need to load the entire training dataset into memory at once. Instead, we can simply load training data in small batches and continuously update our classifier. Therefore, $\mathrm{NBC}++$ can easily use large sizes of $k$-mers for training. In this experiment, we use 5 -fold cross-validation to evaluate the effects of $k$-mer size on taxonomic classification accuracy. Figure 2 shows the average species level accuracy as a function of k-mer size (the standard deviation is not significant thus removed from the visualization). We split our training and testing data on genome level for 5-fold cross-validation, i.e., we evaluate our classifier on reads simulated from genomes/strains that are not used in training phase. Due to cross-validation, the labels of those genomes/strains used for testing may or may not have shown up in the training data (see Additional file 2 - Histogram of the number of genomes per species). And there is a chance that the testing reads are simulated from genomes that are unknown to the classifier (the species level label is unknown to the classifier).

To obtain upper levels of taxonomic classification, the species-level of classification results of known testing reads are "traced back" from the species label using the NCBI taxonomic database [2]. Therefore, we do not need to train the NBC classifier at upper levels such as genus and phylum. All classifications are done at the species level and then "traced back" to upper levels. Figure 3 shows the average accuracy on 6 taxonomic levels: species, genus, family, order, class and phylum, as a function of k-mer size evaluated on known testing reads. Note that species level accuracy is around $84 \%$ and the genus level accuracy is around $92 \%$. As the taxonomy level of classification increases, the accuracy

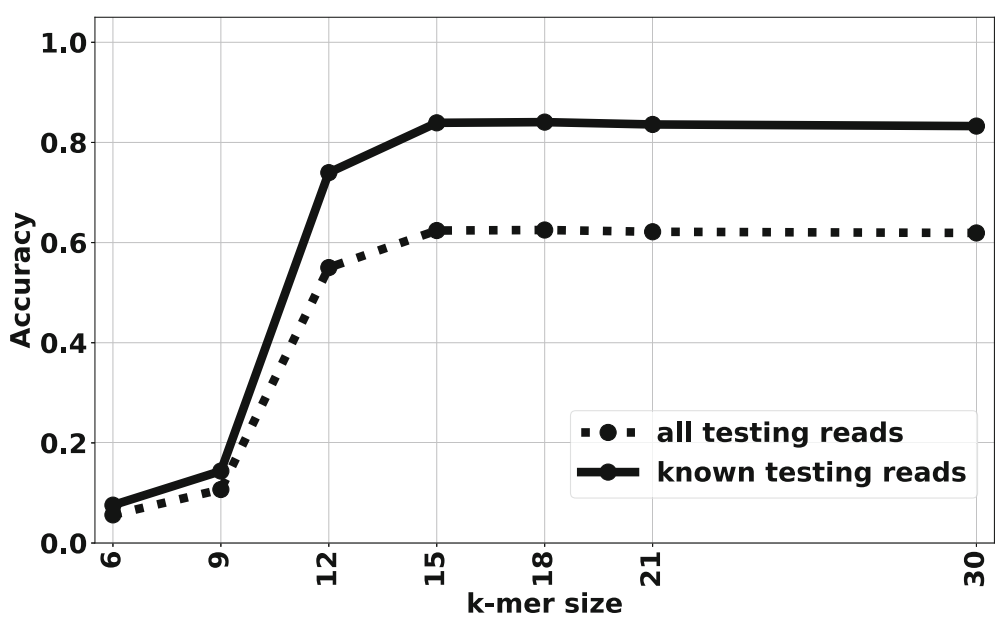

Fig. 2 The species-level NBC++ accuracy vs. k-mer size 


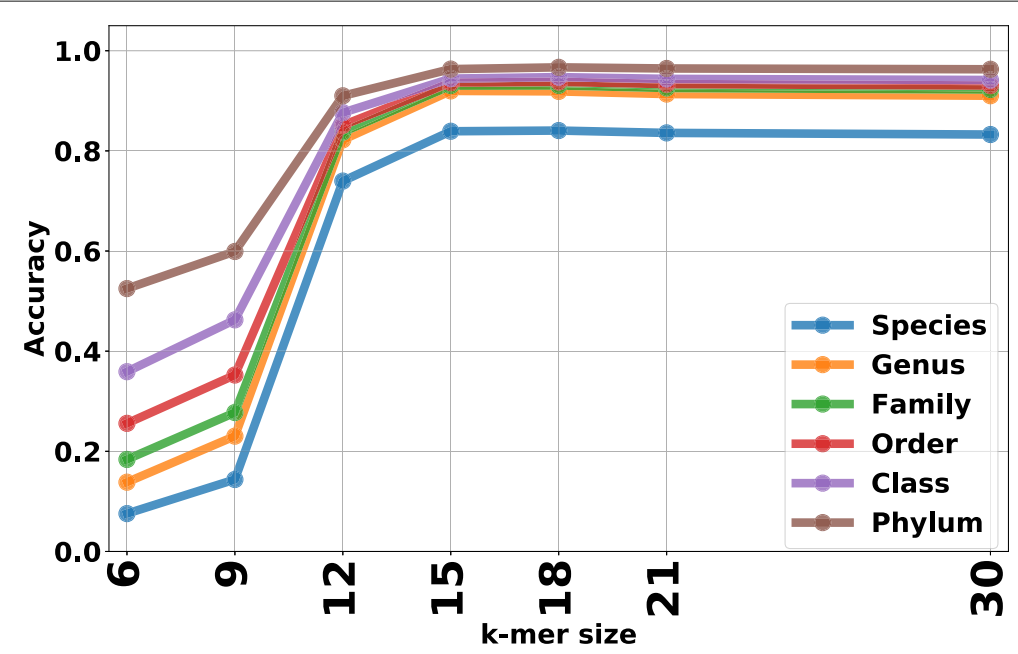

Fig. $3 \mathrm{NBC}++$ accuracy on different taxonomic levels vs. k-mer size. Each read is classified on the species level, and higher-level taxonomic results are calculated by "trace back" through the NCBI phylogenetic tree

increase accordingly. This indicates that although $\mathrm{NBC}++$ can misclassify some testing reads to a wrong species, it may be correct on genus/class/phylum/etc level.

\section{$\mathrm{NBC}++$ taxonomic classification performance}

To evaluate $\mathrm{NBC}++$ while also demonstrating it on a real dataset, we designed experiments to simulate sequences being added yearly to the NCBI bacterial database. We parsed the GCF assembly summary file as it is in March 2nd $2019^{3}$ for the release date of all latest completed genomes. Then the training genomes per year are organized accordingly (see Additional file 3 - RefSeq Bacterial genomes published every year for detailed information).

In Fig. 4, the species-level classification accuracy is calculated for reads simulated from the early-2019 database, with new genomes being sequenced and adding/updating species to the training database each year (the average accuracy and standard deviation per species for 5-fold cross-validation experiment are shown in Additional file 4 - The average accuracy per species from 1999 to 2019 and Additional file 5 - The standard deviation of the accuracy per species from 1999 to 2019). From Fig. 4, we can see a trade-off of more knowledge improving classification (because the class is now known) as opposed to a classifier getting "confused" between classes (as more and perhaps similar classes are added). As more data are being added, the known-species classification accuracy goes down but reaches a stable level (yellow curve), but this loss in "known" performance is dwarfed by the fact that overall accuracy (blue curve) is a function of classes that are "known" (green curve). More specifically, in 2002, as more genomes are added, NBC++ begins to misclassify some species since more are in the database, resulting in a drop to a little over $80 \%$ accuracy by 2005; however, the species that it is classified to has near $90 \%$ accuracy of being from the correct genera (see Fig. 5). This loss in performance is overshadowed when we look at the effect of the database version when using all current taxa. The overall accuracy (blue curve) is a function of the amount of taxa known at that year, and as we know more, the accuracy increases. Note that in 2019, due to cross-validation, the percentage 


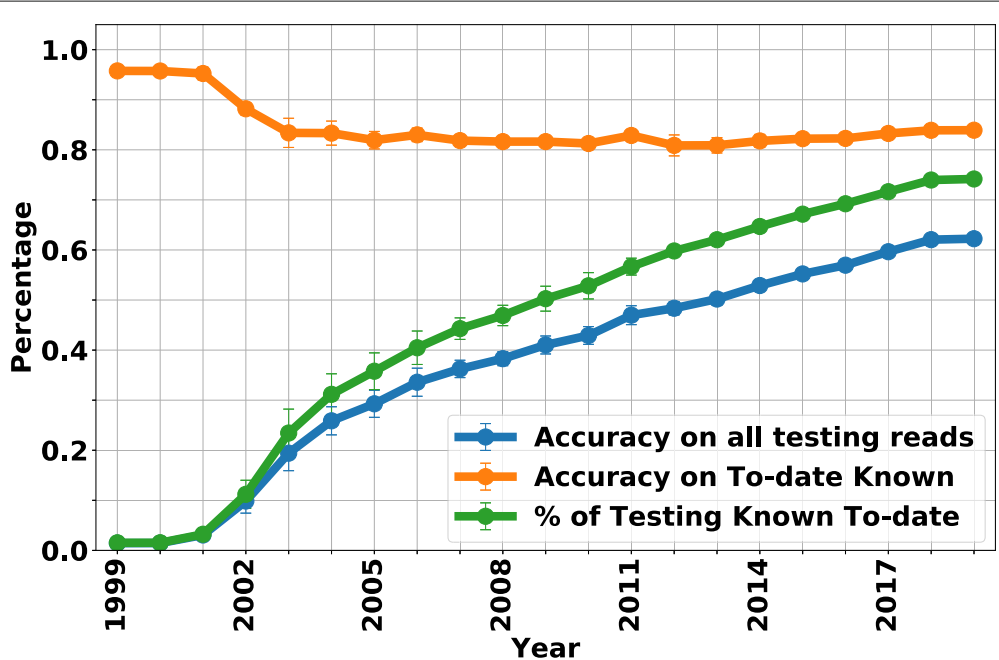

Fig. 4 NBC++ species-level accuracy is evaluated on simulated reads from different species. The $x$-axis represents each year, where the training database is only the genomes added/updated that year. We show that the accuracy on reads from known species up-to-that-year has more than $80 \%$ accuracy and has a drop as more closely related species are added (yellow curve). The accuracy on all testing species (blue curve) increases as a function of the percentage of reads from to-year known species (green curve). Therefore, the accuracy is only as good as the knowledge in our database. If for example, we only have knowledge of 3 species (which was the case in 1999), the accuracy is poor since it is testing on the thousands of species known today (see the Additional file 1 - The number of taxonomic labels per year: Figure A)

of known testing reads is still less than $80 \%$, since some testing reads are from species that are not included in the training (see "Evaluating NBC on a larger dataset: $k$-mer size vs. accuracy" section). As a result, the accuracy on all testing reads in 2019 is less than the accuracy on 2019-known testing reads. To be specific, due to our 5-fold cross-validation, there are many species with less than 5 examples of a species, and therefore, it is marked as unknown if it is not in the training set, resulting in a little over 60\% accuracy by 2018 . This shows that even if a species is known, it still doesn't have enough examples to fully train the diversity of that species. Overall, the incremental implementation can gain the valuable information added per year.

Figure 5 shows the accuracy of $\mathrm{NBC}++$ (assigned on the species-level) for genomes known to the year that is classified (solid) and on all testing reads (dotted) on 6 taxonomic levels (predictions on taxonomic levels higher than species are inferred from the species level predictions). From the figure we can see that as more genomes are added, there is ambiguity confusing the classifier, but this is small compared to the fact that as more data are known, the better the classification become. For a curious exercise, we fitted these curves and predicted when we would achieve 95\% accuracy on all 2019 species/phylum, which was 2057 for species and 2032 for phylum. However, as we know, there will be novel genomes being added in that time, so optimistically, we hope that we could know $95 \%$ of bacterial species in 100-200 years, while knowing all bacterial phyla is emergent.

Figure 6 shows the training time of $\mathrm{NBC}++$ on the database. We compare the $\mathrm{NBC++}$ incremental learning feature with the non-incremental learning setting (shown as NBC in Fig. 6). Although the full training time of traditional NBC is over 16 hours with 15 threads for data up to 2018, the incremental version using all new 2018 genomes to update the existing model from 2017 would only take $\sim 5$ hours with 15 threads. In 2019 , we only 


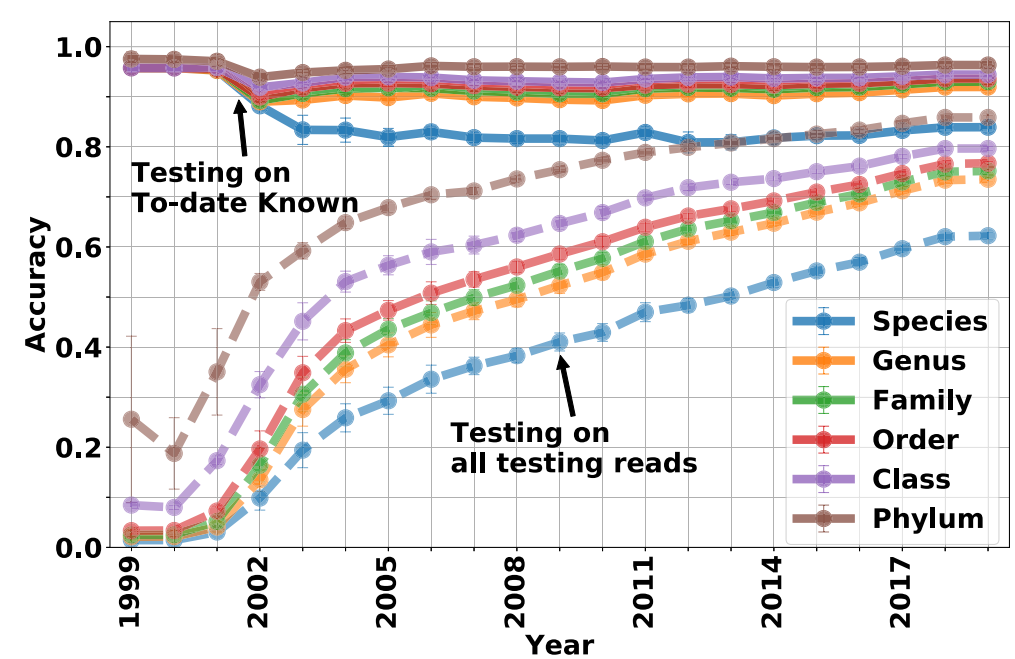

Fig. 5 NBC++ accuracy on all taxonomic levels is evaluated on simulated reads from different species. The accuracy of NBC (assigned on the species-level) on genomes known to-the-year are shown in solid lines and for the entire current set of testing reads is shown in dashed lines. As more genomes are added, there is ambiguity confusing the classifier (most notably on the species level), but this performance loss is small compared to the fact that as more data are known, the better the performance

have new genomes until March 2nd. Therefore, the update time is significantly smaller for $\mathrm{NBC}++$ whereas $\mathrm{NBC}$ has to reprocess the whole database again and results in a waste of computation time.

\section{Evaluation of $\mathrm{NBC}++$ on a healthy gut sample taxonomic profiling results over time}

Nasko et al. [16] uses various experiments to show that Kraken (and derivatives like Bracken) are able to classify more organisms as the RefSeq database grows. Here, we show

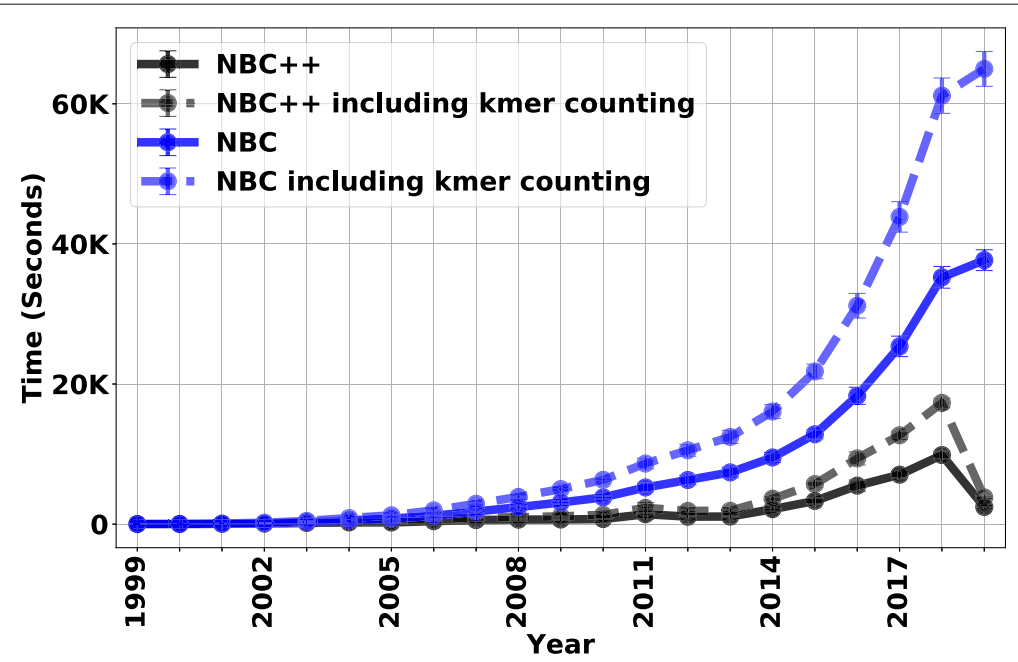

Fig. 6 NBC Incremental learning classifier (NBC++) training/update time is evaluated by the same simulated dataset. This figure shows the update time for incremental learning method (in blue) and traditional full update (NBC) method (in black). The different components of the algorithm are shown - the time that the core NBC algorithm takes (solid) vs. that time plus the average time per genome for Jellyfish to count k-mers (dashed). We can see that the k-mer counting alone can start to add a significant amount of computation when recomputing the entire training dataset 
that NBC++'s classification results change as the database size grows. Note that while $\mathrm{NBC}++$ does not mark anything as "unclassified" (although it has been previous explored in $[39,40])$, its classifications should get more accurate with more data. To demonstrate how up-to-date knowledge affects results, we design real NCBI-over-time experiments to show how the results of $\mathrm{NBC}++$ can be influenced by the training data.

We demonstrate using a time-progressing NCBI training database for NBC to classify a fixed human fecal (a.k.a. gut) sample (SRA ID: SRS105153), which is the same sample used in Fig. 5b in Nasko et al. [16]. For comparison, the NCBI taxonomic classification of this sample can be found online ${ }^{4}$. We also train 5 models using all RefSeq bacterial genomes in NCBI. In Nasko et al., 9 versions of the RefSeq database are used, but the exact results are not discussed - only the level of taxonomy that could be resolved and how many reads remained unclassified. In our experiments, we use the NCBI RefSeq bacterial genomes using 5 database divisions and show how the classifications change over time (with $\mathrm{NBC}++$ labeling everything). The first division is composed of genomes added during 1999, the 2nd division are genomes added during 2000 to 2004 (version 2004), the 3rd division are genomes added during 2005 to 2009 (version 2009), the 4th division are genomes added during 2010 to 2014 (version 2014), and the 5th division are genomes added during 2015 to 2019 (version 2019), respectively. The composition change on different taxonomic levels are evaluated for each of these divisions. Figures 7 and 8 show the predicted bacteria relative abundance of the gut sample over time on the genus and phylum levels, respectively (with species and order levels in the appendix). Taxa with lower than 5\% relative abundance are moved to either "Others: Old" (dark blue, top of the bar) or "Others: New" category (light blue, bottom of the bar) category depending on whether the taxa are recently added or were added in previous years. The training database version that contains the taxa shown in the bar plots are labeled on the bars.

As shown in Figs. 7 and 8 (see also Additional file 6 - Profiling results change over time on species level and Additional file 7 - Profiling results change over time on order level), the profiling results are influenced by the version of training data. For example, in 1999, there are only 4 genomes used to train the database. Therefore, the results in Fig. 8 are drastically skewed and give poor results, with Chlamydiae dominating the gut and no representatives from Bacteroidetes/Firmicutes. As soon as these phylum are introduced by 2004, a great portion of those previously misassigned are assigned to the newly added phyla (and most likely better classifications). We do note that $\mathrm{NBC}++$ is overassigning to Proteobacteria (the amount of Proteobacteria is too high for a healthy gut), due to its over-representation in the training database. However, the percentage of Proteobacteria is steadily decreasing as the amount of training database genomes are getting better, especially with recent additions genomes from Bacteroides/Clostridium/etc. (the proportion being assigned to Bacteroides doesn't significantly increase until the most recent database version, shown in Fig. 7) that are known to be prevalent in gut samples. NBC's classifications are biased by training representation, demonstrating the need for up-to-date knowledge to get the most accurate Bacteroidetes/Firmicutes balance.

As we can see on the genus level, in Fig. 7, the amount of classifications being assigned to newly added genera decrease (although more genera are being added now than in the past, see Additional file 1 - The number of taxonomic labels per year: Figure H). Similar trends

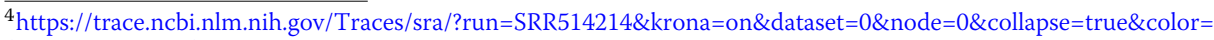
false \&depth $=10 \&$ font $=11 \&$ key=true 


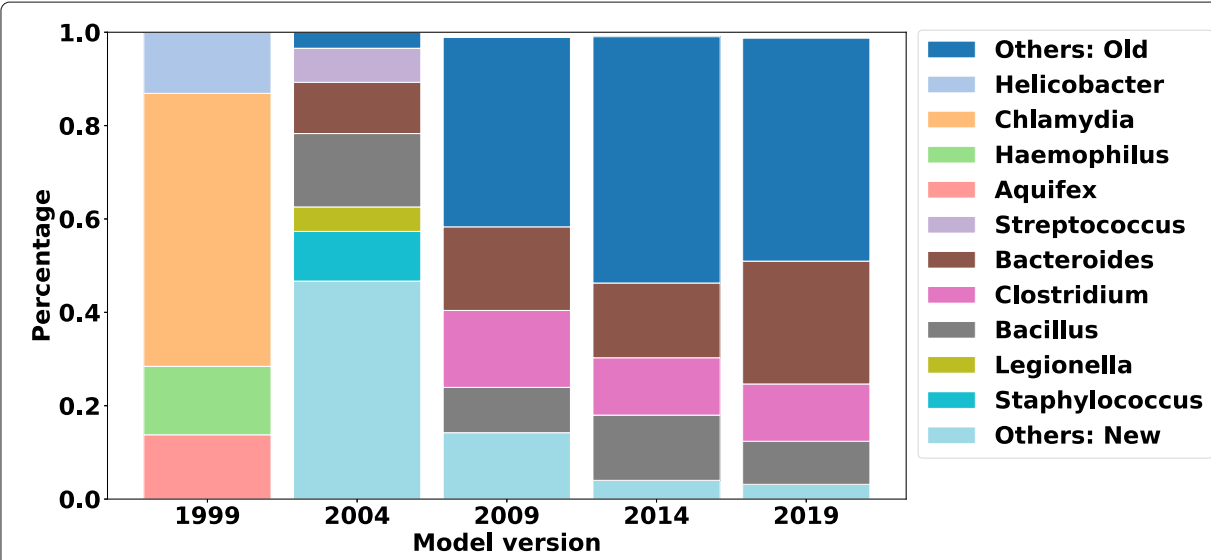

Fig. 7 Profiling results change over time on Genus level: NBC++ classifiers trained on different years are evaluated on a real human fecal sample (SRA ID: SRS105153) on the genus level. The figure shows that the predicted composition of the sample changed over time and is influenced by the training data. Taxa with lower than 5\% relative abundance is moved to either "Others: New" category or "Others: Old" category depending on whether the taxa is recently added in the past 5 years or added in previously

are seen on the species and order levels (see Additional file 6 - Profiling results change over time on species level and Additional file 7 - Profiling results change over time on order level). After 2009, the top three genera (Fig. 7) in the gut sample remain the same Bacteroides, Clostridium, and Bacillus (although not always in the same percentage ranking). In fact, it is surprising that the percentage of Bacteroides significantly increases with new knowledge from the past 5 years, demonstrating that there are Bacteroides genomes being added that are very important to classification of this gut. Specifically, on the species level, when Bacillus ovatus is introduced in the 2019 version (see Additional file 6 - Profiling results change over time on species level), many reads get assigned to it, since it is well-known in the gut [41]. However, as described in “Mistakes: current implementation's tendency to classify to well-represented species classes," section the over-representation of Clostridium botulinum is biasing some of the Clostridium classifications.

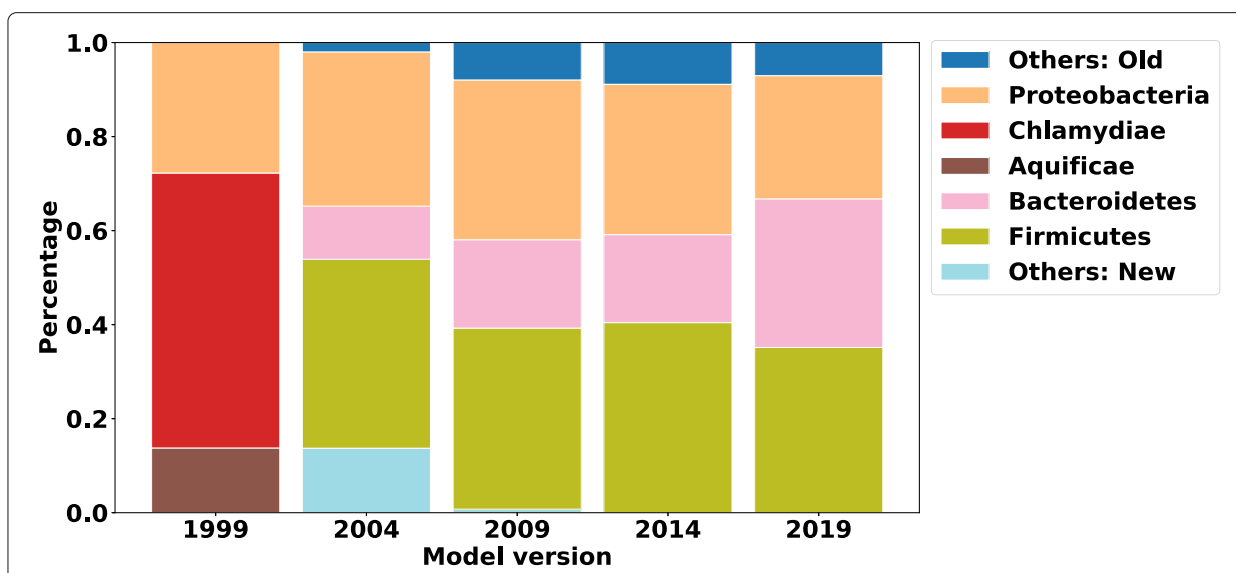

Fig. 8 Profiling results change over time on Phylum level: NBC++ classifiers trained on different years are evaluated on a real human fecal sample (SRA ID: SRS105153) on the phylum level. The figure shows that the predicted composition of the sample changed over time and is influenced by the training data. Taxa with lower than $5 \%$ relative abundance is moved to either "Others: New" category or "Others: Old" category depending on whether the taxa is recently added or added previously 
To further explore how the predicted community composition evolves over time, we calculated the Bray-Curtis dissimilarity between profiling results from consecutive models we trained in this section, i.e., the 1999 and 2004 models; 2004 and 2009 models; 2009 and 2014 models; and 2014 and 2019 models, on different taxonomic levels as shown in Fig. 9. In this analysis, we consider all the taxonomic labels instead of grouping low frequency ones into the "other" bin.

From this figure, we can see, in general, the dissimilarity between community compositions decreases over time. However, we do observe that the dissimilarity scores increase from 2009\&2014 to 2014\&2019. Take the Phylum level as an example, the 2009\&2014 dissimilarity between profiling results is 0.096 which is lower than $2014 \& 2019$ dissimilarity. In fact, as shown in Fig. 8, the relative abundance of predicted Bacteroidetes read (around 32\% of total reads in 2019 profiling result) is $0.4 \%$ lower in 2014 than in 2009. But in 2019 , the abundance is increased by $68.9 \%$ compared with 2014 . One important bacterium found in the gut is B. ovatus, which commonly dominates Bacteroides-rich gut samples [41]. This vital and most likely abundant species being added after 2014 has a drastic effect on the classifications and increases most levels' Bray-Curtis similarity. As more key species are added to RefSeq (e.g. ones that are abundant and play important roles), the classifier will be able to perform profiling more accurately.

\section{Discussion}

In the "Results" section, we show that an incremental learning classifier has the following advantages over the traditional non-incremental version:

- incremental learning can help when a computer has limited memory and cannot process an entire large training dataset at-once - by incrementally processing small batches of the training data at a time.

- the classifier can keep up with the latest knowledge (and associated accuracy gains) efficiently without the cost of a full retrain and therefore reduce the computation time needed for updating the classifier.

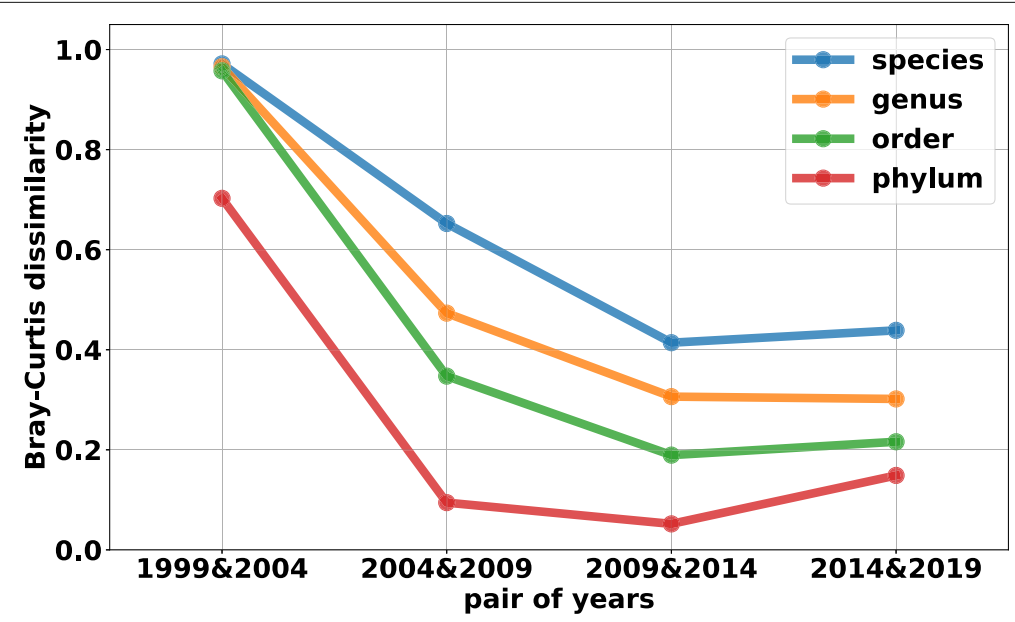

Fig. 9 Bray-Curtis dissimilarity between profiling results over time We use the Bray-Curtis dissimilarity to measure the differences between profiling results in different years on different taxonomic levels. The $x$-axis represents a pair of profiling results the Bray-Curtis dissimilarity is based on. For example, 2009\&2014 means that the Bray-Curtis dissimilarity is calculated between 2009 and 2014 profiling results 
In this section, we discuss the cost of the classifier updates in a broader practical scenario and the classification mistakes that the NBC classifier makes.

\section{Monetary analysis of reduced computation in NBC++}

There are two options to keep our model up-to-date that are discussed in this paper. The first option is to train a model from scratch whenever an updated model is needed. This option requires the users to maintain a database to store the old data or download both new and old data from a remote database, e.g., NCBI database, because training a model from scratch requires both old and new data. And the second option is to update the model with new data only. This option requires the model to be stored in the system for future updates. However, in the best case scenario for the first option, we don't need the access to the model between updates, therefore we don't have to pay for the storage of the model for option 1. Since we need to pay for the raw data storage in option 1 and for the model storage in option 2, we can assume that the storage cost of two options are the same (usually it is not the case because the model size is often smaller than the raw data because model can be considered as a form of abstraction of the raw data). Then the only cost is the computational cost for model update. According to Amazon Web Services (AWS), a "m5.8xlarge" EC2 computing node with 32 cores and 128GB memory costs 1.536 dollars per hour ${ }^{5}$. If it takes 16 hours and 5 hours for option 1 and 2 to run respectively. Option 2 can save 16.896 dollars for only 1 update. If the model is updated daily, at least 6167.04 dollars can be saved without considering the increase of training time when more and more data are added to the database.

Mistakes: current implementation's tendency to classify to well-represented species classes We note that this NBC implementation has certain defects - which may afflict other classifiers as well. This NBC implementation is biased towards calling class labels that have a large abundance of genomic examples. As a case study, we look at mistakes at the phylum level, arguably the most serious ones, which has a 4\% error rate. In the 2019 database, Proteobacteria has 6975 representative genomes while Firmicutes is the next most-represented and has 2925 representatives. Because the algorithm classifies at the species level and then traces up the tree to resolve upper levels, any species that has more occurrences of a $k$-mer as opposed to another species will have an advantage. So, the more genomes that represent a species (or as we sometimes say "examples of that species"), the more likely rare $k$-mers may occur in this particular class (which may accommodate some nucleotide variation).

This biasing of rare $k$-mers can have great impact on the misclassifications that NBC makes, as we can see in Additional file 8 - Misclassified reads - phylum level, which is one fold (out of 5) of the phylum level misclassified reads. In this fold (1/5 of the entire data), there were 7285 misclassified reads on the phylum level out of 186300 reads (96\% accuracy). 2663 out of the 7285 (over 36\%) misclassifications, tended to classify reads as Clostridium botulinum (taxid:1491). This is a problem that becomes even more evident on our test on a real sample (see "Evaluation of $\mathrm{NBC}++$ on a healthy gut sample taxonomic profiling results over time" section). One outstanding example (in the phylum misclassified spreadsheet) is the Buchnera aphidicola (taxid: 9) species, which had 506 
sequences misclassified by NBC, 363 of them classified to C. botulinum, 93 classified to Bacillus cereus/thurigiensis and another 30 to $C$. difficile, all well-represented species in NCBI. As a reference point, there are 62 genome representatives of $B$. aphidicola while there are 242 genome representatitves of $C$. botulinum, 295 representatives of $C$. difficile, and close to similar amount of B. cereus/thurigiensis. What is apparent, is that when some of these sequences are BLASTed (one detailed example in "Case example of B. aphidicola and C. botulinum misclassification" section), they share identical stretches of nucleotides up to 18 bases long with essential C. botulinum protein sequences that have similarity to essential/fundamental proteins such as $\mathrm{ABC}$ transporters, two-component sensor histidine kinases, etc. This shows some important protein sub-domains that are conserved between these organisms. However, due to the single nucleotide mutations that cause these sequences to be unique and prevalence of these subdomains across phyla, these types of sequences are causing NBC to bias its classification towards species that have more representation in the database. This is an important issue to be explored to improve NBC in the future and highlights the importance of keeping up-to-date training data in classifiers and good representation in the database.

\section{Case example of B. aphidicola and C. botulinum misclassification}

Take the following read as an example:

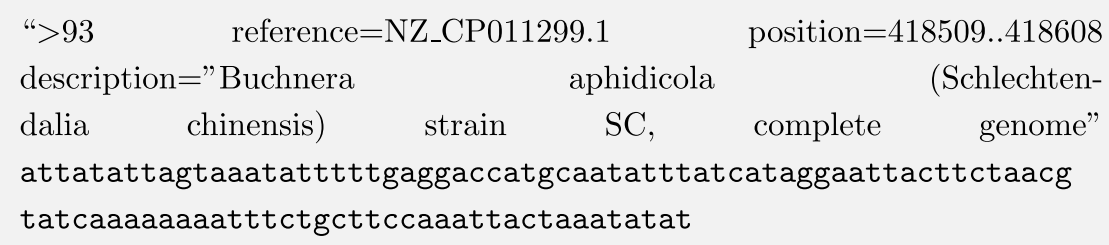

This sequence shares 11 15-mers with the B. aphidiciola (taxid:9) that were trained on for the first fold (see Additional file 9 - 15-mer shared with B. aphidiciola (taxid: 9)), while it shares 18 15-mers with C. botulinum (taxid:1491) (see Additional file 10 - 15-mer shared with C. botulinum (taxid:1491)). While many of the 18 kmers in C. botulinum had lower probability accounting for the fact that $C$. botulinum had $13 \times$ the amount of nucleotides in its class than $B$. aphidiciola, the fact that 7 more unique $k$-mers were found, was substantial enough to tip the classification towards C. botulinum. As a comparison, the B. aphidicola Schlechtendalia chinensis sequence was blasted against other $B$. aphidicola strains and also against $C$. botulinum. The queries match to the B. aphidicola sequence with similarities of ( $58 / 59 \%$ for long stretches and $80-90 \%$ identities for short stretches), for other strains of B. aphidicola excluding strain Schlechtendalia chinensis in Additional file 11 - Blast result for B. aphidiciola (taxid: 9). Similar similarities are found for the blasts of the B. aphidicola query against $C$. botulinum seen in Additional file 12 - Blast result for C. botulinum (taxid:1491). (If the figures only yield an incomplete picture for the reader, we also have search strategies (Additional file 13 - Blast search strategies for B. aphidiciola (taxid: 9), Additional file 14 - Blast search strategies for C. botulinum (taxid:1491)) available to explore this blast). The BLAST results show that even BLAST has slight difficulty distinguishing these sequences. 


\section{Conclusion}

In this paper, we show that new genomes added to the NCBI RefSeq bacterial genome database are exponentially growing. We demonstrate that supervised classification is drastically improving each year with updated NCBI Refseq genome data. By processing simulated reads and a real metagenomic gut sample, we show that new additions to the database change results significantly and have yet to show signs of convergence (i.e. the training database has enough species diversity and example genomes per species to classify samples consistently). Therefore, it is very important to keep the training model updated and that the update process be efficient as possible. We demonstrate that we can achieve efficiency by a proof-of-concept incremental $\mathrm{NBC}++$ taxonomic classifier. By "incrementalizing" taxonomic classifiers, we show that species can be updated/added to the database without the cost of reprocessing the existing database. Our simulation shows that (1) no accuracy is lost compared to the non-incremental NBC implementation and (2) over time, the classification accuracy is a function of the amount of taxa known. Overall, the incremental implementation can gain the valuable information added per year at a fraction of the cost - our example shows that by updating yearly, the 2018 training time would only be 1/4th of the full training time of non-incremental version.

\section{Methods}

\section{Experimental dataset}

In our experiments, we use the NCBI Reference Sequence (RefSeq) bacterial genome database and taxonomic tree [2]. Genomes labeled as "complete Genome" in assembly_level field and "latest" in version_status field are downloaded. Genomes are labeled by their species level label. The genomes are split into 5 folds by random. Testing reads are then simulated from the genomes in 5 folds. 100 reads are simulated from each genome by Grinder [42] with no error. The model will be trained on 4 out of 5 folds and tested on the leftover fold. That is to say, we train on some strains from a species and test on other strains from the same species. In fact, due to this 5 -fold cross-validation, some species with smaller amount of genomes will not show up in training process. Therefore, it is marked as unknown if it is not trained on when evaluate the performance of the classifier. All genomes and testing reads are processed by Jellyfish [43] to count the k-mers in each file as naïve Bayes taxonomic classifier takes the abundance of k-mers as features. For our incremental taxonomic classification experiment, we further separate each fold based on the genomes release year in the assembly summary. Therefore for each year, we have 5 folds and we can train and update the model every year based on which folds the model was originally trained on. For the dynamic of taxonomic profiling experiment, we use all our training genomes as the training data and classify a human fecal sample (SRA ID: SRS105153). We train 5 models in temporal order to show the profiling result change. The initial model is trained by complete Bacteria genomes division 1 (genomes added during 1999), then model 2 is obtained by updating model 1 with training data division 2 (genomes added during 2000 to 2004, model 3 is updated by division 3 (genomes added during 2005 to 2009, followed by model 4 with division 4 (genomes added during 2010 to 2014) and finally model 5 is updated by division 5 (genomes added during 2015 to 2019) from model 4. The composition change on different taxonomic levels are evaluated. 


\section{Naïve Bayes taxonomic classifier}

Our method $(\mathrm{NBC}++)$ is based on a previous non-incremental taxonomic classifier [7]. We then expand the classifier to incrementally update itself efficiently and produce reliable classification results. The core of our classifier is a naïve Bayes (NB) classifier. NB classifier exploits Bayes rule to tackle classification problems which assumes independent features. In essence, NBC's premise is to maximize the (log)-likelihood of observations given each potential class, $P\left(x \mid y_{i}\right)=\prod_{j=1}^{K} P\left(x_{j} \mid y_{i}\right)$, where $x_{j}$ is the $j$ th feature, and $y_{i}$ is label of $i$ th class. The NBC then computes the discriminant function $Q$ (a function of the posterior probability) for each class $y_{i}$ for each observation $x$ as $Q\left(y_{i} \mid x\right)=P\left(y_{i}\right) P\left(x \mid y_{i}\right)$, and chooses the class label for which $Q\left(y_{i} \mid x\right)$ is the largest. In our application, we did not include the prior probability in final likelihood computation because we expect all species equally likely to be observed and don't know which environment or species prior probabilities to expect. Authors now show that having prior information about species likelihood of occurrence are useful [44]. We compute the probability of observing a k-mer given a species via Laplacian smoothing which defined by the following formula:

$$
P\left(x_{j} \mid y_{i}\right)=\frac{f\left(x_{j} \mid y_{i}\right)+1}{\sum_{j=1}^{K} f\left(x_{j} \mid y_{i}\right)+K}
$$

where $f\left(x_{j} \mid y_{i}\right)$ is the total number of a k-mer, $x_{j}$, observed in all training genomes with species level $y_{i} . K$ is the total number of unique $\mathrm{k}$-mers for a given size $\mathrm{k}$ and $K=4^{k}$. The Add- 1 smoothing avoids $P\left(x_{j} \mid y_{i}\right)=0$ when $f\left(x_{j} \mid y_{i}\right)=0$. Then, for a testing reads, $\mathbf{x}$, the posterior probability for a species $y_{i}$ can be obtained by:

$$
p\left(y_{i} \mid \mathbf{x}\right)=\frac{\prod_{j=1}^{K} p\left(x_{j} \mid y_{i}\right)}{\sum_{i=1}^{N} p\left(y_{i}\right) \prod_{i=1}^{K} p\left(x_{j} \mid y_{i}\right)}
$$

where $x_{j}$ is one of the k-mer observed in testing reads $\mathbf{x}$ and $K$ is the total number of unique $\mathrm{k}$-mers observed in the testing reads. There are $N$ species in the training dataset and $p\left(x_{j} \mid y_{i}\right)$ is the probability of observing a k-mer given a species. The prediction is made by taking the argmax of all posterior probabilities. In our implementation, we compute Eq. 2 in log-space. For example, the numerator of $\prod_{j=1}^{K} p\left(x_{j} \mid y_{i}\right)$ in log space becomes the sum of log-probabilities: $L\left(\mathbf{x} \mid y_{i}\right)=\sum_{j=1}^{K} \log \left(p\left(x_{j} \mid y_{i}\right)\right)$.

Traditional NBC implementations require the entire labeled training data to update the model each time when new data are available. Our implementation computes Eq. 1 only for the added or updated species. The log-space version of Eq. 2 is still computed for each "read query".

\section{Incremental naïve Bayes classifier}

The incremental implementation of naive Bayes classifier is quite straightforward. When new sequences are available, we can compute their k-mer of frequency table. Then $P\left(x_{j} \mid y_{i}\right)$ is updated based on the label. For example, if a new genome is from one of the existing classes, then, the frequency of k-mer $x_{j}$ is updated and $P\left(x_{j} \mid y_{i}\right)$ is updated. If the genome is from a novel class, we create a new class in the model and the $P\left(x_{j} \mid y_{i}\right)$ is the frequency of $x_{j}$ divided by the total number of k-mers. In our implementation, $\mathrm{NBC}++$ performs incremental learning in a number of seamless features that allows it to save on work already performed in the past. By structuring trained models into multiple separate files for each class, new classes can be automatically added when encountered 
by the training crawler. In this case, updating the model by a new class is as fast as training on that new class alone. The retraining penalty incurred by incorporating new classes into existing models is eliminated in this way. And adding new data to existing classes is also trivial. By storing some partial data alongside pre-computed priors in its save files, $\mathrm{NBC}++$ can seamlessly expand a class in just the time it takes to train on the number of additional reads being appended to the existing database. This eliminates the need to retrain on sequences which are already part of the database, and greatly improves performance when there is a need to frequently update our database with new information.

Our algorithm can also be extended to allow taxonomic labels merging in the future. For example, species A is merged to species B in NCBI RefSeq database, we can first update the frequency of $x_{j, B}$ by $x_{j, B}^{t+1}=x_{j, B}^{t}+x_{j, A}^{t}$ where $x_{j, B}^{t+1}$ refers to the new frequency of k-mer $j$ of species $B$. And then corresponding conditional probabilities can be updated accordingly.

\section{Scalability of NBC++}

In addition to incremental learning, $\mathrm{NBC}++$ introduces a set of improvements meant to optimally leverage the computational resources at its disposal. $\mathrm{NBC}++$ supports multi-threaded operation, making both training and classification faster by parallelizing independent operations such as working on multiple classes or reads simultaneously. By using a command-line parameter (-t [threads] ), the user can specify the number of threads made available to $\mathrm{NBC}++$. The program will then attempt to run as many parallel instances as threads it has available, and load training/testing data into the available memory for all the threads. The scalability of the previous naïve Bayes classifier is improved. $\mathrm{NBC}++$ can now work within a pre-set memory limit. The program will automatically adjust how many reads it loads into memory, dynamically creating multiple "batches" of various sizes, trying to fit as many as possible in one cycle. This is a sub-optimal but necessary operating mode, as dataset sizes often exceed available RAM. The performance penalty of this process in the case of classification is derived from the repeated iterations through the training database - repeated for each batch. The trade-off is to load and unload the same training classes in/from memory multiple times, because we need to classify multiple batches of reads. We describe the detailed implementation below.

One way in which $\mathrm{NBC}++$ facilitates computational scalability is by automatically separating reads into batches, depending on the amount of memory the user decides to use. By using the $-\mathrm{m}$ [size] argument, the user can instruct $\mathrm{NBC}++$ to keep all batches under the specified memory cap, thus allowing the user to train and classify large datasets on a variety of systems, getting optimal performance on each run without the need for users to manually adjust batch contents. This is particularly helpful when reads in the database have uneven sizes, which would normally make them difficult to manually add to a batch containing a fixed number of reads.

To demonstrate this behavior, consider a scenario where the program is given 510 sequences (of greatly varying lengths) and the memory is capped to 32GBs. Figure 10 depicts how the program would handle batching in this case - the total size of the dataset of reads is $127 \mathrm{GBs}$, so $\mathrm{NBC}++$ begins loading the first 160 into memory. When it detects the next read would exceed its memory cap, the batch totaling 31.7GBs is released for processing. Once processed, the sequences are unloaded from memory and the process 


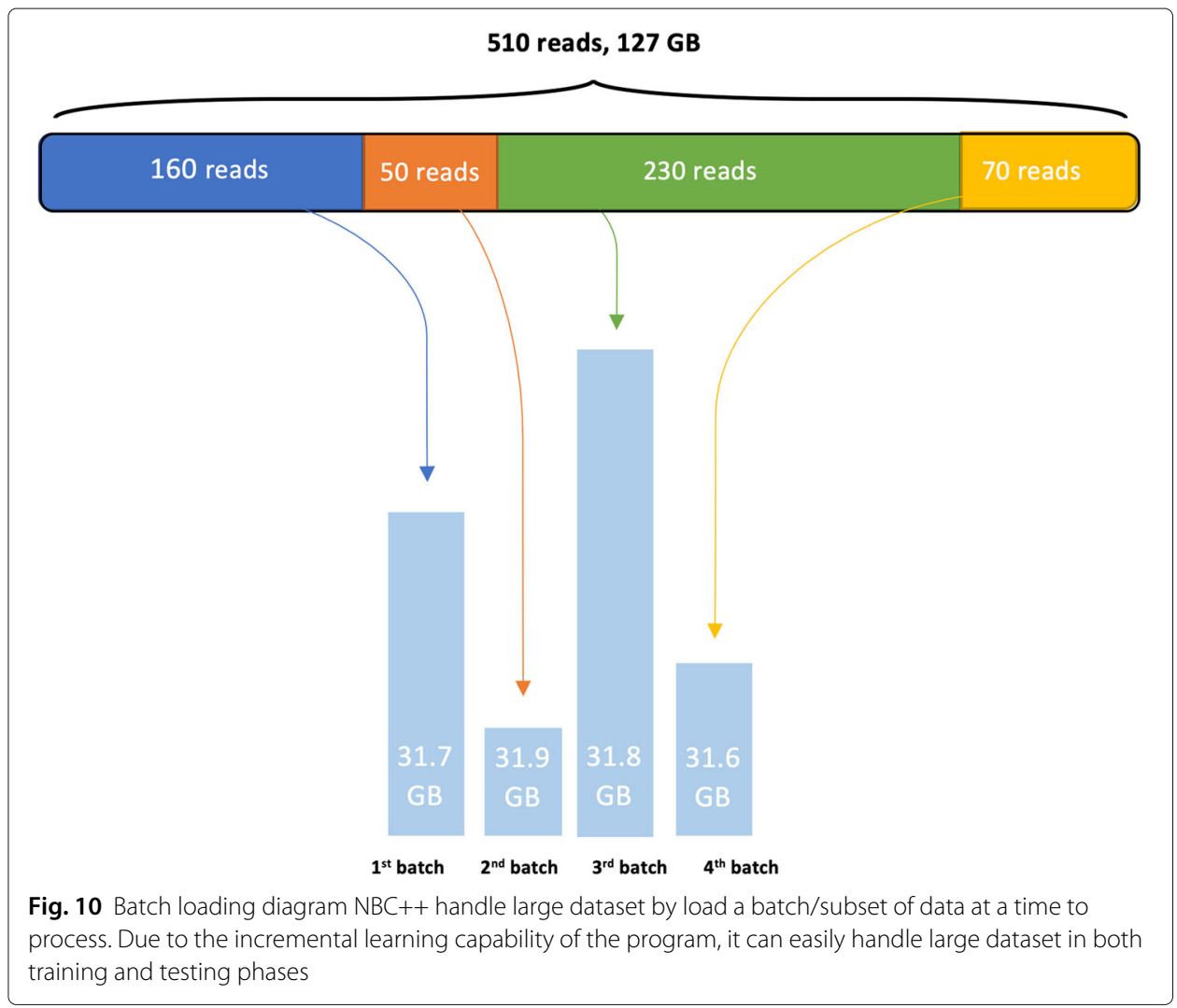

restarts, loading the next batch of sequences until the memory capacity is reached (this time, 50 larger reads take up 31.9GBs). Note that class savefiles will also be loaded for each thread in addition to the loaded reads, therefore the memory cap is only used to dynamically adjust batch sizes.

An additional argument, $-\mathrm{n}$ [nbatch] is provided as a manual override for the number of reads to include in a batch. This argument is not compatible with the memory cap option and should only be used if the user prefers to process a fixed number of reads at a time, rather than trying to use all available memory optimally.

We also allow for multi-threaded computation through the -t [threads] argument, which prompts $\mathrm{NBC}++$ to create worker threads that will concurrently handle one class each. This option is independent of the memory cap, which means that users should allow for some additional memory to be used by each thread in loading their training class savefiles: total_memory $=$ read_memory_cap + n_threads $\times$ savefile_average_size.

With these two options enabled, NBC++ will first create a batch of reads that fit within the provided memory cap. The program will then create the specified number of threads, loading one class savefile for each and processing in parallel all reads within the batch. When we've finished processing all the reads in the batch, the class savefile is unloaded and the next class is read from disk. The thread will then begin to process reads from the beginning. The batch is discarded when this operation has ran for all existing classes, in which case the program will begin creating a new batch and repeating the process until the entire dataset is processed. 
The multi-threaded computation along with memory cap is very useful in computing cluster environment. We use a case study here to illustrate the usage. Suppose we allocated 16 cores and a total of $64 \mathrm{~GB}$ memory resources to run $\mathrm{NBC}++$. Then, the value we pass to - $t$ [threads] would be 15 since we need 1 core for master process and the rest of 15 can be used for multi-threading. The memory cap should be considered as the memory available solely for testing reads. Given we have 16 cores and 64 GB memory, we have $4 \mathrm{~GB}$ per core. If the maximum size of training class savefile (class object of a species derived from training data) is $2 \mathrm{~GB}$. Then $2 \mathrm{~GB}$ per core are available for testing. To be safe, we don't include the memory for master core for testing reads. Then the total amount of memory for testing can be set to $2 \times 15=30 \mathrm{~GB}$. Therefore, we pass $30 \mathrm{~GB}$ to $-m$ [size] parameter. In this way, we manage to balance the loading of both save files (training) and testing data in memory.

\section{Supplementary information}

Supplementary information accompanies this paper at https://doi.org/10.1186/s12859-020-03744-7.

Additional file 1: The number of taxonomic labels per year. The number of updates in the NCBI bacteria genome database on six taxonomic levels, namely, species, genus, family, order, class and phylum. We have a figure for "Accumulative number of updates per year" per taxonomic level (A, B, C, D, E, F) and a figure for "compared with last year, the number of new updates per year" per taxonomic level $(G, H, I, J, K, L)$.

Additional file 2: Histogram of the number of genomes per species. We plot the histogram of the number of genomes per species every year. The figures show that many species have only one genome. We designed our experiment so that we can train a model on some genomes of a species and then evaluate the model with other genomes of the same species. Therefore, when we train on those species, we don't have a testing genome to simulate testing reads from. And when we do simulate testing reads from those genomes, they don't exist in the training set. To this end, we evaluate our model with two metrics: accuracy for all reads and accuracy for known reads (reads from known species).

Additional file 3: RefSeq bacterial genomes published every year. This table shows the release year, the organism name and taxonomic ID of completed genomes in RefSeq Bacterial assembly summary.

Additional file 4: The average accuracy per species from 1999 to 2019. The table shows the average accuracy per species for 5-fold cross-validation.

Additional file 5: The standard deviation of the accuracy per species from 1999 to 2019. The table shows the standard deviation of the accuracy per species for 5 -fold cross-validation.

Additional file 6: Profiling results change over time on species level. The NBC incremental learning classifiers trained on different years are evaluated on a real human fecal sample (SRA ID: SRS105153) on species level. The figure shows that the predicted composition of the sample changed over time and is influenced by the training data. Taxa with lower than $5 \%$ relative abundance is moved to either "Others: New" category or "Others: Old" category depending on weather the taxa is recently added or was added in previous section.

Additional file 7: Profiling results change over time on order level. The NBC incremental learning classifiers trained on different year are evaluated on a real human fecal sample (SRA ID: SRS105153) on order level. The figure shows that the predicted composition of the sample changed over time and is influenced by the training data. Taxa with lower than 5\% relative abundance is moved to either "Others: New" category or "Others: Old" category depending on weather the taxa is recently added or was added in previous section.

Additional file 8: Misclassified reads - phylum level. We trace our species classification results back to phylum level and most of the reads are assigned to the correct phylum level taxonomic labels. This table shows the reads that got misclassified in phylum level.

Additional file 9: 15-mer shared with B. aphidiciola (taxid: 9). 15-mer shared between the case example read in "Case example of B. aphidicola and C. botulinum misclassification" section and fold-1 training data for B. aphidiciola (taxid: 9).

Additional file 10: 15-mer shared with C. botulinum (taxid:1491).15-mer shared between the case example read in "Case example of B. aphidicola and C. botulinum misclassification" section and fold-1 training data for C. botulinum (taxid:1491).

Additional file 11: Blast result for B. aphidiciola (taxid: 9). Blast result for the case example read in "Case example of B. aphidicola and C. botulinum misclassification" section against B. aphidiciola (taxid: 9) exclusing strain Schlechtendalia chinensis.

Additional file 12: Blast result for C. botulinum (taxid:1491). Blast result for the case example read in "Case example of B. aphidicola and C. botulinum misclassification" section against C. botulinum (taxid:1491).

Additional file 13: Blast search strategies for B. aphidiciola (taxid: 9). The Blast search strategies for the case example read in "Case example of B. aphidicola and C. botulinum misclassification" section 
against B. aphidiciola (taxid: 9) exclusing strain Schlechtendalia chinensis. The file ends in ASN and the reader can also open it as a normal text file.

Additional file 14: Blast search strategies for C. botulinum (taxid:1491). The Blast search strategies for the case example read in "Case example of B. aphidicola and C. botulinum misclassification" section against C. botulinum (taxid:1491). The file ends in ASN and the reader can also open it as a normal text file.

\section{Abbreviations}

NBC: Naïve Bayes classifier; NBC++: Our "incrementalized" version of naïve Bayes classifier; NCBI: National center for biotechnology information; RefSeq: The NCBI reference sequence database

\section{Acknowledgements}

This work was partially supported by the Extreme Science and Engineering Discovery Environment (XSEDE), which is supported by National Science Foundation grant number ACl-1548562. Specifically, it used the Bridges system, which is supported by NSF award number ACl-1445606, at the Pittsburgh Supercomputing Center (PSC) [45, 46]. This work was partially supported by Drexel's University Research Computing Facility (URCF).

\section{Authors' contributions}

ZZ: Formal analysis, Methodology, Supervision, Validation, Visualization, Writing - original draft \& review \& editing AC: Software Development, Formal analysis, Methodology, Validation, Writing - original draft GR: Conceptualization, Funding acquisition, Resources, Supervision, Writing - original draft \& review \& editing. All authors have read and approved the manuscript.

\section{Funding}

This project is funded by an NSF I/UCRC grant \#1650431 through an industry-university center, the Center for Visual and Decision Informatics (CVDI), and NSF grant \#1936791. Under CVDI, Becton, Dickinson and Company (BD) supported us for this project. While the BD funded stipend and tuition, they had no role in study design, data collection and analysis, decision to publish, or preparation of the manuscript.

\section{Availability of data and materials}

We use the NCBI Reference Sequence (RefSeq) bacterial genome database and taxonomic tree [2] as of March 2nd 2019. The bacterial genome data is downloaded from the NCBI website according to the GCF assembly summary file as it is in March 2nd 2019 download from ftp://ftp.ncbi.nlm.nih.gov/genomes/refseg/bacteria/assembly_summary.txt. The taxonomic tree is downloaded from ftp://ftp.ncbi.n/m.nih.gov/pub/taxonomy/taxdump.tar.gz as it is in March 2nd 2019. The real human fecal sample used in our experiment is publicly available with SRA ID: SRS105153. Our NBC++ software is open-source and can be obtained through: https://github.com/EESI/Naive_Bayes.

\section{Ethics approval and consent to participate}

Not applicable

\section{Consent for publication}

Not applicable

\section{Competing interests}

The content of this paper is related to a pending patent application entitled "Multi-temporal Information Object Incremental Learning Software System" filed in 2018/3/2.

\section{Author details}

${ }^{1}$ Ecological and Evolutionary Signal-process and Informatics (EESI) Lab, Department of Electrical and Computer Engineering, Drexel University, Market Street, Philadelphia, US. ${ }^{2}$ Department of Computer Science, Drexel University, Market Street, Philadelphia, US.

Received: 29 February 2020 Accepted: 8 September 2020

Published online: 21 September 2020

\section{References}

1. Zynda GJ. Exponential growth of NCBI genomes. http://gregoryzynda.com/ncbi/genome/python/2014/03/31/ncbigenome.html. Accessed 07 June 2019.

2. Sayers EW, Barrett T, Benson DA, Bryant SH, Canese K, Chetvernin V, Church DM, DiCuccio M, Edgar R, Federhen S, Feolo M, Geer LY, Helmberg W, Kapustin Y, Landsman D, Lipman DJ, Madden TL, Maglott DR, Miller V, Mizrachi I, Ostell J, Pruitt KD, Schuler GD, Sequeira E, Sherry ST, Shumway M, Sirotkin K, Souvorov A, Starchenko G, Tatusova TA, Wagner L, Yaschenko E, Ye J. Database resources of the national center for biotechnology information. Nucleic Acids Res. 2008;37(suppl_1):5-15. https://doi.org/10.1093/nar/gkn741.

3. Benson DA, Karsch-Mizrachi I, Lipman DJ, Ostell J, Sayers EW. GenBank. Nucleic Acids Res. 2008;37(suppl_1):26-31. https://doi.org/10.1093/nar/gkn723.

4. Kyrpides NC, Hugenholtz P, Eisen JA, Woyke T, Göker M, Parker CT, Amann R, Beck BJ, Chain PSG, Chun J, Colwell RR, Danchin A, Dawyndt P, Dedeurwaerdere T, DeLong EF, Detter JC, De Vos P, Donohue TJ, Dong X-Z, Ehrlich DS, Fraser C, Gibbs R, Gilbert J, Gilna P, Glöckner FO, Jansson JK, Keasling JD, Knight R, Labeda D, Lapidus A, Lee J-S, Li W-J, MA J, Markowitz V, Moore ERB, Morrison M, Meyer F, Nelson KE, Ohkuma M, Ouzounis CA, Pace N, Parkhill J, Qin N, Rossello-Mora R, Sikorski J, Smith D, Sogin M, Stevens R, Stingl U, Suzuki K.-i., Taylor D, Tiedje JM, Tindall B, Wagner M, Weinstock G, Weissenbach J, White O, Wang J, Zhang L, Zhou Y-G, Field D, Whitman WB, Garrity GM, Klenk H-P. Genomic encyclopedia of bacteria and archaea: Sequencing a myriad of type strains. PLoS Biol. 2014;12(8):1001920. https://doi.org/10.1371/journal.pbio.1001920. 
5. Cullen CM, Aneja KK, Beyhan S, Cho CE, Woloszynek S, Convertino M, McCoy SJ, Zhang Y, Anderson MZ, Alvarez-Ponce D, Smirnova E, Karstens L, Dorrestein PC, Li H, Gupta AS, Cheung KKW, Powers JG, Zhao Z, Rosen GL. Emerging priorities for microbiome research. Front Microbiol. 2020;11:136.

6. Wetterstrand KA. DNA sequencing costs: data from the NHGRI Genome Sequencing Program (GSP). https://www. genome.gov/about-genomics/fact-sheets/DNA-Sequencing-Costs-Data. Accessed 07 June 2019.

7. Rosen GL, Reichenberger ER, Rosenfeld AM. NBC: the Naïve Bayes Classification tool webserver for taxonomic classification of metagenomic reads. Bioinformatics. 2011;27(1):127-9. https://doi.org/10.1093/bioinformatics/ bta619.

8. Wood DE, Salzberg SL. Kraken: ultrafast metagenomic sequence classification using exact alignments. Genome Biol. 2014;15(3):1-12. https://doi.org/10.1186/gb-2014-15-3-r46.

9. Ames SK, Hysom DA, Gardner SN, Lloyd GS, Gokhale MB, Allen JE. Scalable metagenomic taxonomy classification using a reference genome database. Bioinformatics. 2013;29(18):2253-60. https://doi.org/10.1093/bioinformatics/ btt389.

10. Segata N, Waldron L, Ballarini A, Narasimhan V, Jousson O, Huttenhower C. Metagenomic microbial community profiling using unique clade-specific marker genes. Nat Methods. 2012;8:811-4. https://doi.org/10.1038/nmeth.2066

11. Menzel P, Ng KL, Krogh A. Fast and sensitive taxonomic classification for metagenomics with Kaiju. Nat Commun. 2016;7:11257.

12. Clarke EL, Taylor LJ, Zhao C, Connell A, Lee J-J, Fett B, Bushman FD, Bittinger K. Sunbeam: an extensible pipeline for analyzing metagenomic sequencing experiments. Microbiome. 2019;7(1):46. https://doi.org/10.1186/s40168019-0658-x.

13. Mclntyre ABR, Ounit R, Afshinnekoo E, Prill RJ, Hénaff E, Alexander N, Minot SS, Danko D, Foox J, Ahsanuddin S, Tighe S, Hasan NA, Subramanian P, Moffat K, Levy S, Lonardi S, Greenfield N, Colwell RR, Rosen GL, Mason CE. Comprehensive benchmarking and ensemble approaches for metagenomic classifiers. Genome Biol. 2017;18(1): 182. https://doi.org/10.1186/s13059-017-1299-7.

14. Sczyrba A, Hofmann P, Belmann P, Koslicki D, Janssen S, Dröge J, Gregor I, Majda S, Fiedler J, Dahms E, Bremges A, Fritz A, Garrido-Oter R, Jørgensen TS, Shapiro N, Blood PD, Gurevich A, Bai Y, Turaev D, DeMaere MZ, Chikhi R, Nagarajan N, Quince C, Meyer F, Balvočiūtè M, Hansen LH, Sørensen SJ, Chia BKH, Denis B, Froula JL, Wang Z, Egan R, Don Kang D, Cook JJ, Deltel C, Beckstette M, Lemaitre C, Peterlongo P, Rizk G, Lavenier D, Wu Y-W, Singer SW, Jain C, Strous M, Klingenberg H, Meinicke P, Barton MD, Lingner T, Lin H-H, Liao Y-C, Silva GGZ, Cuevas DA, Edwards RA, Saha S, Piro VC, Renard BY, Pop M, Klenk H-P, Göker M, Kyrpides NC, Woyke T, Vorholt JA, Schulze-Lefert P, Rubin EM, Darling AE, Rattei T, McHardy AC. Critical assessment of metagenome interpretation — a benchmark of metagenomics software. Nat Methods. 2017;14:1063-71.

15. Loeffler C, Karlsberg A, Martin LS, Eskin E, Koslicki D, Mangul S. Improving the usability and comprehensiveness of microbial databases. BMC Biology. 2020;18(1):1-6. https://doi.org/10.1186/s12915-020-0756-z.

16. Nasko DJ, Koren S, Phillippy AM, Treangen TJ. RefSeq database growth influences the accuracy of k-mer-based lowest common ancestor species identification. Genome Biol. 2018;19(1):1-10. https://doi.org/10.1186/s13059-0181554-6.

17. Zhao Z, Rosen G. Multi-temporal Information Object Incremental Learning Software System. Google Patents. 2018. https://patents.google.com/patent/US20180253529A1/en.

18. Taninpong $\mathrm{P}$, Ngamsuriyaroj $\mathrm{S}$. Incremental naïve Bayesian spam mail filtering and variant incremental training. In: 2009 Eighth IEEE/ACIS International Conference on Computer and Information Science; 2009. p. 383-7.

19. Salperwyck $C$, Lemaire $V$, Hue $C$. Incremental weighted naive bays classifiers for data stream. In: ECDA; 2013.

20. Lu J, Yang Y, Webb Gl. Incremental discretization for naïve-bayes classifier. In: International Conference on Advanced Data Mining and Applications; 2006. p. 223-38, Springer.

21. Zhao Z, Rollins J, Bai L, Rosen G. Incremental author name disambiguation for scientific citation data. In: 2017 IEEE International Conference on Data Science and Advanced Analytics (DSAA); 2017. p. 175-83. https://doi.org/10. 1109/DSAA.2017.17.

22. Kochurov M, Garipov T, Podoprikhin D, Molchanov D, Ashukha A, Vetrov D. Bayesian incremental learning for deep neural networks. arXiv preprint arXiv:1802.07329. 2018.

23. Castro FM, Marín-Jiménez MJ, Guil N, Schmid C, Alahari K. End-to-end incremental learning. In: Ferrari V, Hebert M, Sminchisescu C, Weiss Y, editors. Computer Vision - ECCV 2018. Cham: Springer; 2018. p. 241-57.

24. Polikar R, Upda L, Upda SS, Honavar V. Learn++: an incremental learning algorithm for supervised neural networks. IEEE Trans Syst Man Cybern Part C Appl Rev. 2001;31(4):497-508. https://doi.org/10.1109/5326.983933.

25. Ruping S. Incremental learning with support vector machines. In: Proceedings 2001 IEEE International Conference on Data Mining; 2001. p. 641-2. https://doi.org/10.1109/ICDM.2001.989589.

26. Zheng J, Shen F, Fan H, Zhao J. An online incremental learning support vector machine for large-scale data. Neural Comput \& Applic. 2013;22(5):1023-35. https://doi.org/10.1007/s00521-011-0793-1.

27. Xu J, Xu C, Zou B, Tang YY, Peng J, You X. New incremental learning algorithm with support vector machines. IEEE Trans Syst Man Cybern Syst. 2018;49(11):2230-41. https://doi.org/10.1109/TSMC.2018.2791511.

28. McDonald D, Xu Z, Hyde ER, Knight R. Ribosomal RNA, the lens into life. Cold Spring Harbor Laboratory Press for the RNA Society. 2015;21(4):692-4. https://doi.org/10.1261/rna.050799.115.

29. Wang Q, Garrity GM, Tiedje JM, Cole JR. Naïve Bayesian classifier for rapid assignment of rRNA sequences into the new bacterial taxonomy. Appl Environ Microbiol. 2007;73(16):5261-7. https://doi.org/10.1128/AEM.00062-07.

30. Lan Y, Rosen $G$, Hershberg R. Marker genes that are less conserved in their sequences are useful for predicting genome-wide similarity levels between closely related prokaryotic strains. Microbiome. 2016;4(1):1-13.

31. Lan Y, Morrison JC, Hershberg R, Rosen GL. POGO-DB?a database of pairwise-comparisons of genomes and conserved orthologous genes; 2014. p 625-32.

32. Altschul SF, Gish W, Miller W, Myers EW, Lipman DJ. Basic local alignment search tool. J Mol Biol. 1990;215(3): 403-10. https://doi.org/10.1016/S0022-2836(05)80360-2.

33. Huson D, Auch A, Qi J, Schuster SC. MEGAN analysis of metagenomic data. Genome Res. 2007;17:377-86. https:// doi.org/10.1101/gr.5969107. 
34. Koslicki D, Zabeti H. Improving minhash via the containment index with applications to metagenomic analysis. Appl Math Comput. 2019;354:206-15.

35. Ounit R, Wanamaker SI, Close TJ, Lonardi S. CLARK: fast and accurate classification of metagenomic and genomic sequences using discriminative k-mers. BMC genomics. 2015;16(1):236.

36. Rojas-Carulla M, Tolstikhin IO, Luque G, Youngblut N, Ley R, Schölkopf B. Genet: Deep representations for metagenomics. arXiv preprint arXiv:1901.11015. 2019.

37. Liang Q, Bible PW, Liu Y, Zou B, Wei L. DeepMicrobes: taxonomic classification for metagenomics with deep learning. bioRxiv. 2019. https://doi.org/10.1101/694851, https://www.biorxiv.org/content/early/2019/07/09/694851. full.pdf.

38. Fiannaca A, Paglia LL, Rosa ML, Bosco GL, Renda G, Rizzo R, Gaglio S, Urso A. Deep learning models for bacteria taxonomic classification of metagenomic data. BMC Bioinformatics. 2018;19(7):198.

39. Rosen GL, Polikar R, Caseiro DA, Essinger SD, Sokhansanj BA. Discovering the unknown: improving detection of novel species and genera from short reads. J Biomed Biotechnol. 2011;2011:495849. https://doi.org/10.1155/2011/ 495849 .

40. Lan Y, Wang Q, Cole JR, Rosen GL. Using the RDP classifier to predict taxonomic novelty and reduce the search space for finding novel organisms. PLoS ONE. 2012;7(3):32491.

41. Kraal L, Abubucker S, Kota K, Fischbach MA, Mitreva M. The prevalence of species and strains in the human microbiome: A resource for experimental efforts. PLoS ONE. 2014;9(5):97279.

42. Angly FE, Willner D, Rohwer F, Hugenholtz P, Tyson GW. Grinder: a versatile amplicon and shotgun sequence simulator. Nucleic Acids Res. 2012;40(12):94. https://doi.org/10.1093/nar/gks251.

43. Marçais $G$, Kingsford C. A fast, lock-free approach for efficient parallel counting of occurrences of k-mers. Bioinformatics. 2011;27(6):764-70. https://doi.org/10.1093/bioinformatics/btr011.

44. Kaehler BD, Bokulich N, McDonald D, Knight R, Caporaso JG, Huttley GA. Species abundance information improves sequence taxonomy classification accuracy. Nat Commun. 2019;10(1):1-10. https://doi.org/10.1101/406611.

45. Towns J, Cockerill T, Dahan M, Foster I, Gaither K, Grimshaw A, Hazlewood V, Lathrop S, Lifka D, Peterson GD, Roskies R, Scott JR, Wilkins-Diehr N. XSEDE: Accelerating scientific discovery. Comput Sci Eng. 2014;16(5):62-74. https://doi.org/10.1109/MCSE.2014.80.

46. Nystrom NA, Levine MJ, Roskies RZ, Scott JR. Bridges: a uniquely flexible HPC resource for new communities and data analytics. In: Proceedings of the 2015 XSEDE Conference: Scientific Advancements Enabled by Enhanced Cyberinfrastructure. New York, NY, USA: ACM; 2015. p. 1-8. https://doi.org/10.1145/2792745.2792775.

\section{Publisher's Note}

Springer Nature remains neutral with regard to jurisdictional claims in published maps and institutional affiliations.

- fast, convenient online submission

- thorough peer review by experienced researchers in your field

- rapid publication on acceptance

- support for research data, including large and complex data types

- gold Open Access which fosters wider collaboration and increased citations

- maximum visibility for your research: over $100 \mathrm{M}$ website views per year

At BMC, research is always in progress.

Learn more biomedcentral.com/submissions 